\title{
Factors Influencing Whether or Not Stroke Patients Are Discharged Home after Rehabilitation
}

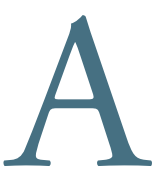

number of factors are associated with likelihood of being discharged home after rehabilitation for stroke:

- High motor function: Stroke patients who were admitted to rehabilitation with greater independence in daily physical activities were six times more likely to be discharged home than patients admitted with less independence.

- Pre-stroke living arrangement: Patients who lived with a spouse or family member prior to their stroke were four times more likely to be discharged home than those who lived alone.

- Age: Patients who were younger than 65 were three times as likely to return home after rehabilitation as those who were 85 or older.

- Sex: Male patients admitted for stroke rehabilitation were 1.3 times more likely to be discharged home than female patients, after all other factors are considered.

- Time to Inpatient Rehabiliation: Patients admitted to rehabilitation within the seven days following their stroke were twice as likely to be discharged home as compared to those admitted 22 days or more post-stroke, independent of level of function at time of admission

- Stroke Related Impairment: Stroke patients admitted to inpatient rehabilitation with a stroke that affected only one side of their body (unilateral involvement) were over twice as likely to be discharged home than those who were admitted with bilateral body involvement.

The type of hospital where a stroke patient received rehabilitation services (specialty versus general) did not have a significant effect on a patient's likelihood of being discharged home.

* Material abstracted from Factors Predicting Discharge Home From Inpatient Rehabilitation After Stroke released by CIHI in May 2009. <http://secure.cihi.ca/cihiweb/dispPage.jsp?cw_ page=PG_2070_E\&cw_topic $=2070 \&$ cw_rel=AR_2891_E. >

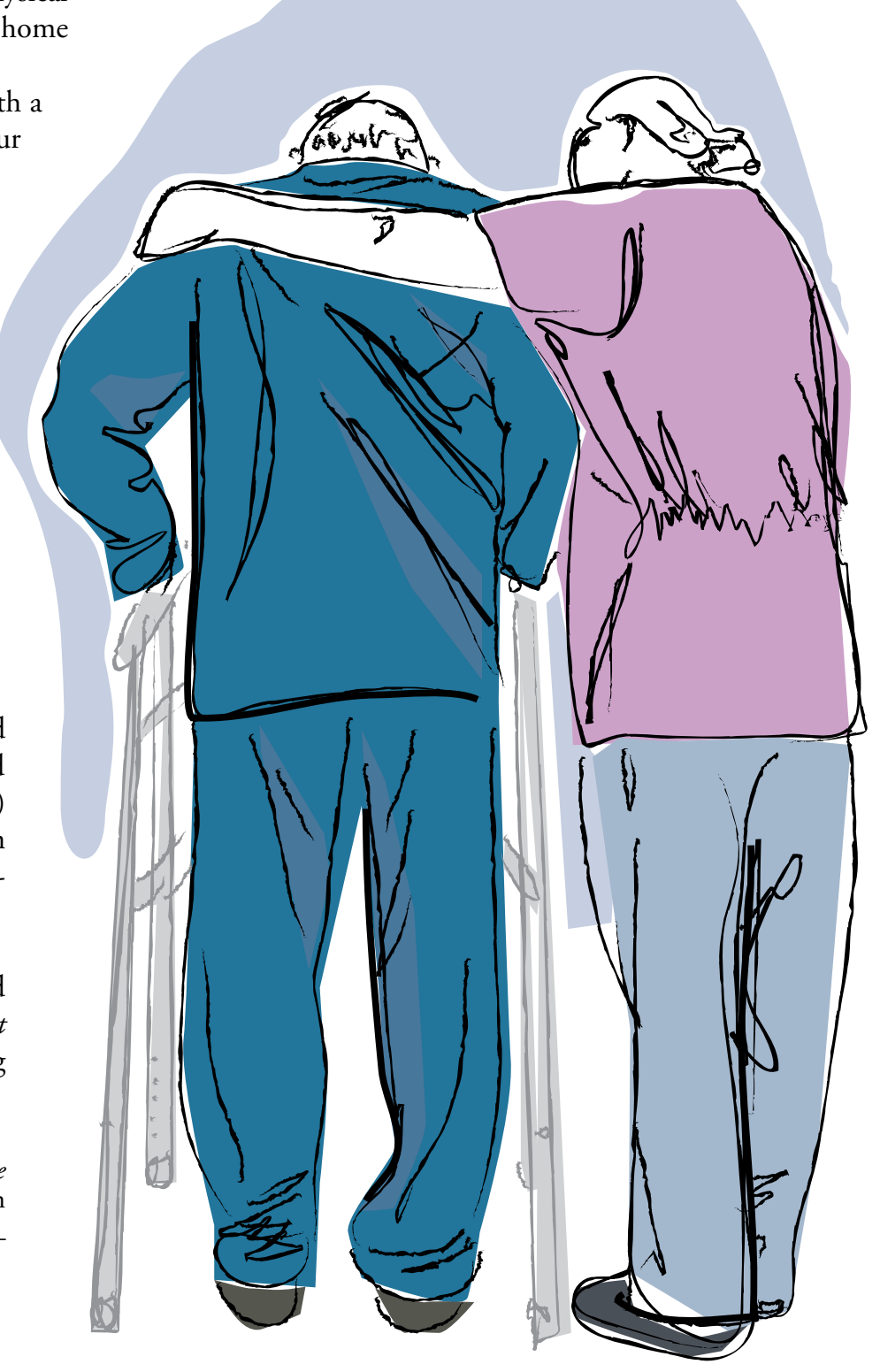

